\title{
Central Nervous System Germ Cell Tumor
}

National Cancer Institute

\section{Source}

National Cancer Institute. Central Nervous System Germ Cell Tumor. NCI Thesaurus.

Code C5461.

A unique group of rare tumors of the central nervous system that affect mainly children and adolescents. Their morphologic and biologic profile corresponds to that of homologous germ cell tumors that arise in the gonads and in other extragonadal sites. Representative examples include: germinoma, embryonal carcinoma, yolk sac tumor, choriocarcinoma, and teratoma. 